\title{
Pratiques et Perceptions d'un Appareil Étatique en Voie de Décentralisation : le Cas du Bénin
}

\section{Christophe Anthoine}

\section{OpenEdition}

12 Journals

Édition électronique

URL : http://journals.openedition.org/apad/534

DOI : 10.4000/apad.534

ISSN : 1950-6929

Éditeur

LIT Verlag

Édition imprimée

Date de publication : 1 décembre 1998

\section{Référence électronique}

Christophe Anthoine, «Pratiques et Perceptions d'un Appareil Étatique en Voie de Décentralisation : le Cas du Bénin », Bulletin de l'APAD [En ligne], 16 | 1998, mis en ligne le 06 octobre 2006, consulté le 08 septembre 2020. URL : http://journals.openedition.org/apad/534; DOI : https://doi.org/10.4000/apad. 534

Ce document a été généré automatiquement le 8 septembre 2020

Bulletin de l'APAD 


\title{
Pratiques et Perceptions d'un Appareil Étatique en Voie de Décentralisation : le Cas du Bénin
}

\author{
Christophe Anthoine
}

1 Tandis que le processus de décentralisation piétine au Bénin ${ }^{1}$, l'École Nationale d'Administration de ce pays et l'Université Libre de Bruxelles (Belgique) se sont associées pour initier un projet d'aide au développement des administrations locales ${ }^{2}$, Il concerne les actuelles circonscriptions urbaines ${ }^{3}$, sur lesquelles portera la prochaine décentralisation, et les instances administratives qui lui sont subordonnées (quartiers et communes). Dans ce cadre, une large enquête est actuellement en cours; elle touchera 6 circonscriptions urbaines (une par département selon le découpage territorial toujours en vigueur) : Bohicon (département du Zou), Kandi (département du Borgou), Lokossa (département du Mono), Natitingou (département de l'Atacora), Ouidah (département de l'Atlantique) et Pobè (département de l'Ouémé), Mis à part des questions techniques relatives aux fonctions des agents de l'administration, elle porte essentiellement sur la perception de la décentralisation future par la population et les élus locaux (maires de commune, chefs de quartier et conseillers des précédents), sur la pratique et la perception des élections locales ainsi que sur les rapports entre administrés et administrations locales,

2 Je voudrais ici exploiter les premiers résultats de cette enquête -je m'appuierai à cette fin sur les enquêtes effectuées à Bohicon (janvier-février 1998), Lokossa (janvier-février 1998) et Ouidah ${ }^{4}$ (juin-juillet 1997)- pour esquisser une 'approche par le bas' ${ }^{5}$ de la décentralisation au Bénin.

Perception par la population d'un projet étatique : la décentralisation

3 Au Bénin, la décentralisation s'inscrit dans une réforme de l'administration territoriale qui, en raison d'accords politiques difficiles sur les structures juridiques qui la régiront, n'est pas encore effective. Décidée lors de la Conférence des forces vives de la nation en février 1990, cette réforme comprend, outre la décentralisation, deux mesures qui doivent l'accompagner : 

territorial et de la décentralisation proprement dite, ont assuré à la réforme territoriale
un écho médiatique notable ${ }^{6}$. Il en a résulté une popularisation du terme 'décentralisation' mais aussi, pour certains, une mécompréhension du concept. En effet, s'il est encore fréquent de rencontrer, généralement parmi les couches les plus défavorisées de la population, des personnes qui n'ont jamais entendu parler de la décentralisation ou qui n'en connaissent que le nom ${ }^{7}$, un nombre non négligeable de Béninois qui sont à même de lui donner un contenu en ont une compréhension erronée ou confuse.

8 Le plus souvent, ils assimilent à la décentralisation les autres aspects de la réforme territoriale. Elle est ainsi régulièrement confondue avec le découpage territorial suite à la publicité faite, sous l'intitulé de la décentralisation, aux aspects les plus épineux de cette question. A Ouidah, l'opposition farouche à la nomination d'Allada comme chef-lieu du nouveau département dont elle doit faire partie est à l'origine d'une nette réticence à l'égard de la décentralisation. Cette dernière étant soit réduite à cette question, qui a bénéficié d'une publicité maximale dans la circonscription, soit perçue comme le vecteur de cette mesure.

9 Une autre source de confusion provient du fait que l'appellation 'commune' glissera à un niveau supérieur de la hiérarchie administrative: les communes actuelles abandonneront leur nom aux circonscriptions urbaines décentralisées pour prendre celui d'arrondissement. Il s'ensuit parfois des interprétations paradoxales de la décentralisation : ainsi beaucoup de Béninois voient en elle une centralisation visant à une compression du personnel (en particulier le moins qualifié) !

Cela dit, quand on s'intéresse à ce qui détermine l'attitude des Béninois ${ }^{8}$ à l'égard de la décentralisation, on se rend compte que l'opinion d'un nombre important d'entre eux est davantage déterminée par la liaison de ce processus à l'État que par le contenu réel des mesures envisagées. Ce qui donne à la décentralisation sa légitimité, ce qui la rend acceptable ou inacceptable par la population, c'est le fait qu'elle est proposée par l'État, qu'elle vient "d'en haut" 9.

11 En effet, la décentralisation n'étant toujours au Bénin qu'un projet - les différentes modalités qui doivent l'organiser restant à définir -, il est bien malaisé de savoir aujourd'hui ce qu'elle sera concrètement et quelles en seront les conséquences pratiques pour les populations, Si l'on ajoute à cela la mauvaise information, ou l'absence d'information, qui règne à son propos au sein de la population, on comprend plus aisément pourquoi l'attitude des uns et des autres vis-à-vis de la décentralisation, qu'elle soit positive ou négative, reflète fondamentalement un positionnement par rapport à l'État. L'État reste le référent ultime, l'interlocuteur absolu du Béninois, comme l'illustrent bien ces propos d'une jeune marchande de Ouidah :

"Tout ce que l'État décide est bon. Est-ce que nous pouvons quelque chose contre notre sort? Le mieux pour nous maintenant est de regarder faire" 10 
La position des Béninois vis-à-vis de la décentralisation peut certes varier en fonction de réalités locales. Mais si, dans l'ensemble, les Béninois sont bien disposés à l'égard de la décentralisation - tout en n'ayant guère d'avis affirmés sur la question -, il transparaît dans leur attitude un sentiment résigné d'impuissance accompagné, pour certains, d'une confiance maintenue 'malgré tout' (à défaut d'alternative ?) dans la bienveillance et la clairvoyance de l'État. Ce sentiment est d'autant plus fort que le niveau de scolarisation est moindre : les Béninois les plus faiblement scolarisés (et c'est en particulier le cas des femmes issues des milieux les plus défavorisés) s'estiment explicitement inaptes à juger l'action de l'État.

13 Il est cependant un aspect de la décentralisation qui inquiète fort la frange de la population pour qui ce processus ne se résume pas à un mot; c'est, on n'en sera pas surpris, la question des moyens financiers dont devront disposer les futures communes décentralisées. En effet, bien ou mal comprise, la décentralisation consiste néanmoins pour les personnes consultées en un retrait de l'État dont elles craignent qu'il se transforme en un abandon. Selon ce point de vue, la décentralisation laisserait alors aux seules nouvelles communes la responsabilité d'assurer le développement local. Or il s'agit là d'une tâche qui nécessite des moyens financiers non négligeables et à laquelle les Béninois accordent la plus grande importance, leurs attentes dans ce domaine portant prioritairement sur un entretien de qualité et le développement de l'infrastructure routière ainsi que, là où elles ne sont pas présentes, l'adduction d'eau et l'électrification ${ }^{11}$. Dans leur esprit, le développement local constitue déjà à l'heure actuelle la fonction principale de l'administration locale (après celle de délivrer des documents administratifs) et ils estiment à ce propos qu'elle l'assume mal ou insuffisamment. Ils redoutent que la décentralisation ait pour effet d'accentuer cette situation, l'État se désengageant davantage tout en n'allouant pas plus de moyens financiers aux entités décentralisées. La population craint par conséquent d'être amenée à assumer seule le développement local, car, quand bien même les Béninois se déclarent prêts à collaborer à la prise en charge de leur destin, d'abord ils conditionnent leur participation à une gestion transparente des efforts de développement et à des résultats visibles, ensuite ils indiquent bien que leur dénuement économique est tel qu'il faut attendre de leur part un soutien davantage moral qu'économique et que, dans ce dernier domaine, il faut envisager une participation en nature (par des prestations gratuites de services) plutôt que monétaire. Aussi, ne voyant pas de quelle façon les entités décentralisées pourraient subvenir à leurs besoins accrus, les Béninois redoutent d'être abandonnés par l'État, dont ils attendent toujours l'intervention, et craignent que la décentralisation ne les livre par conséquent à eux-mêmes.

14 Pour une part, ces résultats de l'enquête ENA-ULB sont comparables à ceux d'une enquête menée en 1992 sous l'égide de la Fondation Konrad Adenauer et portant également sur la perception par la population de la décentralisation ${ }^{12}$ (Séhouéto 1992).

Dans les deux cas sont apparus une confusion entre la décentralisation et le découpage territorial (ce dernier étant une source de conflit), la crainte d'un abandon de l'État (notamment dans le domaine de l'enseignement) et d'énormes attentes vis-à-vis de ce dernier.

Par contre, si en 1992 comme aujourd'hui, la notion de participation populaire demeure un élément clé de la décentralisation dans l'esprit des Béninois, à présent ils ne l'envisagent plus uniquement sous l'angle d'une participation à la définition des 
priorités locales et aux processus de prise de décisions, mais également sous celui d'une participation à la charge économique de la décentralisation qui, en 1992, n'était même pas évoquée ${ }^{13}$. Il faut cependant restreindre la portée de cette constatation pal' le fait que, comme nous l'avons vu, on peut s'interroger sur cette volonté de participation : ne se limite-t-elle pas à une déclaration de bonnes intentions?

Le caractère identitaire des propos liés à la décentralisation est un autre point de divergence entre les deux enquêtes. Il semble nettement moins marqué actuellement qu'en 1992, mais peut-être ne s'agit-il que d'une différence d'appréciation. On notera en outre que, sur cette question, il peut y avoir d'importantes variations d'une localité à une autre (Séhouéto 1992: 51 ; lors de notre enquête, cette dimension identitaire était fort présente à Ouidah en raison du conflit avec Aliada mais très peu à Lokossa -qui est de création récente- et Bohicon) et que ce facteur peut expliquer cette divergence entre les deux enquêtes.

Enfin, si la notion d'un contrôle des actes posés dans le cadre d'une administration décentralisée n'avait pas été relevée dans les propos recueillis en 1992, elle apparaît aujourd'hui dans l'exigence d'une transparence dans la gestion et de résultats visibles que la population place comme préalables à toute participation.

Depuis 1992, la construction politique de la réforme territoriale, et avec elle sa couverture médiatique, a considérablement progressé. A l'époque de l'enquête de la Fondation Konrad Adenauer, la discussion de la décentralisation était essentiellement technique et confinée aux allées du pouvoir. Désormais, même si la contradiction entre le discours de l'État (participation de la population à la gestion de la chose publique) et ses actes (discussion de la décentralisation au seul plus haut niveau, entre experts et politiciens) relevée alors par M. Séhouéto (1992: 53) reste présente, la discussion de la décentralisation a néanmoins pris un tour plus politique et la forme d'un débat public, largement couvert par les médias, avec l'examen et le vote au parlement des lois qui donneront à la réforme territoriale son contenu. On ne peut donc plus parler d'un déficit d'information sur les différents aspects de la décentralisation au même titre qu'en 1992, la population étant aujourd'hui davantage informée et de façon plus précise (la réforme territoriale se faisant elle-même plus précise au fur et à mesure de sa construction politique). Il n'en demeure pas moins qu'un effort reste à faire pour fournir aux populations une information qui soit à la fois complète, détaillée et accessible (j'entends par là compréhensible par l'ensemble de la population) sur ces questions. De ce point de vue, il sera peut-être nécessaire de dispenser une information plus personnalisée, qui tiendra compte, d'une part, des réalités propres au public auquel elles s'adressent et, d'autre part, des modes d'intelligibilité et des schémas interprétatifs avec lesquels il les recevra.

Stratégies populaires de recours a l'administration locale

Si l'étude de la perception populaire du projet de décentralisation politique au Bénin nous éclaire sur la position de la population à ce sujet, l'analyse des modes d'interaction des Béninois avec leur administration locale sous sa forme actuelle et de l'appréhension qu'ils en ont nous instruit, quant à elle, sur le contexte dans lequel la décentralisation s'insèrera. A cet égard, on verra que certaines des stratégies élaborées par la population pour traiter avec l'administration auront des conséquences originales sur la décentralisation une fois celle-ci mise en pratique.

21 Les Béninois ont peu de contacts avec leurs administrations locales et l'idée qu'ils se font de leurs fonctions se réduit aux raisons pour lesquelles ils interagissent avec elles. 
Pour eux, le rôle principal de l'administration locale, c'est de fournir des documents administratifs, au premier rang desquels se place la carte d'identité ${ }^{14}$, les actes de naissance et les légalisations (notamment d'actes de vente) venant ensuite ${ }^{15}$.

La plupart d'entre eux conviendront en outre qu'il revient également à l'administration locale, et à la circonscription urbaine en particulier, d'assurer le développement de l'entité qu'elle administre et d'y percevoir des taxes. Il n'est pas clair, dans l'esprit des Béninois, d'où l'administration locale tire les ressources nécessaires pour assurer le développement de la circonscription. S'ils supposent que les sommes perçues à titre de frais (pour la délivrance de documents) et de taxes devraient être destinées à cet usage, ils doutent fort que ce soit le cas. C'est manifestement l'État central qui est supposé pourvoir au financement du développement local, aidé à l'occasion par la population qui arrive à recueillir des fonds, via des souscriptions, quand ils sont destinés à des objectifs précis et fédérateurs (construction d'une école, adduction d'eau, électrification, etc.). D'une manière générale, la population soupçonne, voire est convaincue, que l'argent qui passe entre les mains de l'administration locale est détourné à des fins personnelles, et cela à l'insu de l'État : ainsi la remise d'un reçu lors de la perception de taxes par les agents d'une circonscription urbaine n'est pas, aux yeux de nos interlocuteurs, la garantie d'une gestion transparente et légale de ces fonds, mais un leurre qui masque en réalité l'accaparement de ces sommes par les administrateurs locaux.

De tels propos indiquent clairement un manque de confiance dans les capacités des responsables administratifs locaux à mener une gestion saine des fonds publics. Pourtant, il ne s'agit peut-être là que de l'expression d'une rhétorique du pouvoir beaucoup plus générale au Bénin, la politique du ventre y étant, comme ailleurs en Afrique, le registre privilégié d'énonciation du politique ${ }^{16}$ (Olivier de Sardan 1996 : 99-100, Banégas $1998: 83-7)$. On verra en effet que la position de la population à l'égard des représentants locaux de l'administration est plus complexe que ne le laissent entendre ces propos.

Si l'obtention de documents est pratiquement l'unique raison pour laquelle la grande majorité des Béninois sont amenés à entrer en relation avec l'administration ${ }^{17}$, ce sont pourtant des démarches auxquelles ils se résolvent rarement et, généralement, en raison d'une obligation ou d'un intérêt à intégrer la sphère formelle de l'État. Ce sera notamment le cas de l'étudiant qui veut passer le baccalauréat, ce qui nécessite de posséder une carte d'identité, ou, moins fréquemment, de l'opérateur économique qui veut se soustraire aux risques du secteur informel. Par exemple, un réparateur de deux roues peut décider d'intégrer la sphère de l'économie formelle en déclarant son activité commerciale auprès de la circonscription urbaine - et en payant les taxes liées à cette existence officielle - afin d'éviter que les agents de cette dernière ne fassent saisir les véhicules qui ne sont pas en règle dans son atelier.

Lorsqu'un Béninois désire obtenir un document auprès de cette administration qui lui est si étrangère, diverses stratégies lui permettent de l'acquérir d'une façon relativement satisfaisante. Le recours à ces stratégies varie selon les lieux et les individus, comme le montrent des exemples tirés des circonscriptions urbaines de Ouidah et de Lokossa.

26 La principale exigence des habitants de Ouidah à l'égard de l'administration porte sur une procédure rapide pour la délivrance de documents. L'obtention d'un document nécessite généralement de se rendre successivement dans différentes instances de 
l'administration locale (quartier, commune, circonscription urbaine). Pour une carte d'identité par exemple, un Béninois doit s'adresser auprès du chef de son quartier pour obtenir un certificat de résidence qu'il devra ensuite faire contresigner par le maire de sa commune - s'il ne réside pas dans la commune où il est né, il devra aussi se rendre auprès du maire de cette dernière pour disposer d'un extrait d'acte de naissance -, avant de pouvoir s'adresser à la circonscription urbaine où tous ces documents lui seront réclamés. Dans chacune de ces instances, il faut, si l'on veut être servi directement et éviter ainsi une perte de temps et d'argent (frais de déplacement), trouver sur place la personne habilitée à délivrer ces documents (c'est-à-dire celle qui dispose du cachet et de la signature). Or cela est plus aisé en ce qui concerne la circonscription urbaine -le lieu de travail des responsables concernés, et le quartier où l'on va voir le chef à son domicile, en soirée -, qu'en ce qui concerne la commune. Dans ce dernier cas en effet, il convient de trouver le maire à la maison communale ; or, l'activité de maire n'étant pas une activité professionnelle - les maires comme les chefs de quartiers ne bénéficient pas d'un salaire, à l'instar des agents de circonscription urbaine, mais d'indemnités ${ }^{18}$-, leur présence à la mairie est intermittente et varie fort d'un maire à l'autre ; le plus souvent un maire occupe la maison communale plusieurs matinées par semaine, mais il arrive régulièrement qu'il doive s'absenter pour des raisons privées ${ }^{19}$. C'est la raison pour laquelle la population préfère généralement que la fonction de maire soit occupée par des retraités (qui n'ont plus d'activités professionnelles limitant leur disponibilité).

Pour accélérer la procédure de délivrance des documents administratifs et satisfaire ce qu'on qualifierait presque d'obsession de la vitesse ${ }^{20}$, les habitants de Ouidah ont couramment recours à diverses stratégies. Ils peuvent "déposer la pierre", c'est-à-dire donner quelque chose au fonctionnaire pour qu'il fasse diligence ${ }^{21}$; il s'agit en quelque sorte d'une procédure d'urgence informelle dont le surcoût est empoché à titre privé par le fonctionnaire sollicité. Ils peuvent aussi chercher un service accéléré auprès de leurs connaissances travaillant dans l'administration - qu'il s'agisse d'un parent, d'un ami ou autre ${ }^{22}$-, ou auprès d'une administration réputée efficace (autrement dit, délivrant rapidement les documents indépendamment de toute intervention personnalisée) plutôt que de s'adresser à l'administration prescrite par la loi.

La loi béninoise détermine en effet l'administration à laquelle les citoyens sont tenus de s'adresser; il s'agit le plus souvent, hormis quelques cas particuliers comme les actes de naissances par exemple, des instances administratives du lieu de résidence. Dans les faits cependant, cette règle n'est contraignante que pour les copies d'actes de naissance (qui doivent nécessairement être demandées dans la commune où a eu lieu la naissance, là où se trouve le registre de naissances), les légalisations d'actes de vente de propriétés foncières (obligatoirement effectuées auprès de l'administration sur le territoire de laquelle se trouve la propriété foncière) et les actes nécessitant un certificat de résidence (délivré par le chef du quartier et nécessairement contresigné par le maire de la commune à laquelle appartient ce quartier). Pour d'autres actes administratifs (légalisations, etc.), rien n'empêche de s'adresser à n'importe quelle administration. Dans ces cas-là, un habitant de Ouidah aura donc tendance à s'adresser à l'administration où il pourra faire intervenir une connaissance en sa faveur ou à celle qui offre un meilleur service que d'autres.

29 Cela dit, même dans les cas les plus contraignants, les Béninois sont en mesure de choisir leur administration. S'ils ont par exemple besoin d'un document qui requiert un 
certificat de résidence, ils peuvent s'appuyer sur la pluralité de leur territorialisation sociale en vue d'obtenir cette pièce le plus rapidement possible. Ainsi, un jeune homme qui avait besoin d'une carte d'identité pour passer son baccalauréat, trouvant la circonscription urbaine de Ouidah trop lente, a préféré demander celle-ci à Cotonou. A cette fin, il s'est fait établir un certificat de résidence auprès du chef d'un quartier de Cotonou où il a l'habitude de fréquenter des membres de sa famille et a ainsi pu mener toutes ses démarches auprès des instances administratives de Cotonou. Un autre habitant de Ouidah, appliquant la même stratégie, s'est fait établir un certificat de résidence non par le chef du quartier où il vit mais par celui du quartier où il travaille parce que ce dernier relève de la commune dont le maire est un ami de sa famille. Ces pratiques s'appuient à la fois sur la faiblesse du contrôle effectué par le chef de quartier pour s'assurer que le demandeur réside bien dans son quartier ${ }^{23}$ et sur la multiplicité des ancrages du demandeur ${ }^{24}$.

Certaines de ces stratégies, comme le fait de s'adresser à une administration en raison de son efficacité intrinsèque ou de jouer sur la multiplicité de sa territorialisation sociale, sont fondées sur une mobilité aisée -propre en particulier à ceux qui se rendent fréquemment à Cotonou, comme les navetteurs qui y logent en semaine pour y travailler ou étudier et rentrent à Ouidah le week-end ${ }^{25}$ - et sur une bonne intelligibilité de l'administration, ce qui est surtout le fait d'individus moyennement ou fortement scolarisés. La pratique qui fait appel à l'ancrage administratif des réseaux sociaux est, quant à elle, fréquente et répandue dans toutes les couches de la population et dépend de la capacité de chacun à construire et mobiliser de tels réseaux.

31 Il est à noter que l'ensemble de ces stratégies vise principalement le niveau intermédiaire de l'administration locale, celui de la commune, qui apparaît de ce point de vue comme son maillon faible. Nous avons déjà souligné que la moindre disponibilité du maire rendait plus difficile l'obtention immédiate d'un document. C'est pourquoi il est intéressant de s'adresser à une maison communale où l'on sait avoir plus de chance d'y trouver le maire que dans une autre, et le maire qui fait preuve d'une plus grande disponibilité est souvent sollicité par des citoyens issus d'autres communes ${ }^{26}$.

Cela peut constituer pour lui un moyen d'augmenter ses ressources privées s'il s'accapare, en tout ou en partie, les sommes payées pour les actes qu'il pose. Mais le plus souvent, les citoyens de Ouidah s'adressent à un maire qu'ils connaissent personnellement et qu'ils peuvent alors aller solliciter à son domicile privé. Nous allons voir que ce dernier procédé, qui est l'une des modalités du recours à l'ancrage administratif des réseaux sociaux, est à l'origine d'une utilisation des élections locales pour réaliser cet ancrage.

Les élections locales comme mode d'ancrage des réseaux sociaux dans l'appareil d'état

33 Le Bénin a connu ces dernières années la mise en place de pratiques visant des buts semblables à ceux de la décentralisation, si l'on veut bien admettre que cette dernière doit principalement "rapprocher l'Administration du citoyen" et permettre "l'exercice du pouvoir de décision par les populations à la base à travers leurs représentants élus" (Dossou $1997:$ 1). C'est tout particulièrement le cas des élections locales au niveau des communes et des quartiers et, dans une nettement moindre mesure, de la nomination par l'État de chefs de circonscription urbaine originaires de celles-ci.

34 Les premières élections locales démocratiques depuis l'ère Kérékou I ont eu lieu en 1991. Après 17 ans de régime marxiste, elles ont représenté pour la population béninoise l'opportunité d'élire leurs représentants dans un contexte politique neuf 
marqué par une volonté de changement; il s'ensuivit que, plutôt que de confirmer systématiquement des titulaires liés à l'ancien régime comme c'était le cas auparavant, elles furent l'occasion d'un choix réel et entraînèrent souvent l'élection de nouveaux représentants locaux ${ }^{27}$.

Ces élections concernaient les postes de maire, de chef de quartier et, de manière plus informelle apparemment, de conseillers de ces derniers. Alors que le mandat de ces élus est officiellement de 5 ans, de nouvelles élections n'ont plus été organisées depuis lors 28 .

Lors des élections de 1991, le degré d'intimité liant un candidat à son électeur a autant déterminé le vote de ce dernier que son aptitude à exercer la fonction à laquelle il était candidat. Ce sont les réseaux qui structurent la société béninoise (parenté, voisinage, parti politique, associations diverses, amitié, etc.) qui ont déterminé ce degré d'intimité. Certes, cette attitude n'est pas spécifique aux élections locales, ${ }^{29}$ mais elle porte ici sur une proximité autrement plus grande que pour d'autres mandats (présidence, parlement), de telle sorte qu'elle influe nettement sur les rapports des individus avec l'élu. En effet, dans ce cas-ci tout particulièrement, l'électeur n'agit pas seulement en vertu d'une logique communautaire. Il cherche aussi, par ce vote, à élire un représentant local vis-à-vis duquel il pourra faire intervenir d'autres types de rapports que ceux d'administré à administrateur.

A Ouidah, ces logiques se sont principalement exprimées sur une base individuelle, chaque électeur (ou groupe restreint d'électeurs) tentant, par son vote, d'ancrer un réseau qui lui est propre dans l'appareil administratif. Pour ce faire, certains de ses habitants n'ont pas hésité à utiliser la pluralité de leur territorialisation sociale, tel cet artiste peintre de 35 ans qui, n'ayant de relations privilégiées avec aucun des candidats à la mairie de la commune où il réside, s'est fait inscrire comme électeur dans la commune de son lieu de travail, pour pouvoir y soutenir un candidat qui lui est proche (en l'occurrence le président de son club de pétanque).

Ce sont ces mêmes logiques qui ont guidé les électeurs de Lokossa, mais elles ont été ici mises en œuvre collectivement, sous l'égide des notables locaux ${ }^{30}$. Comme nous allons le voir, on en est ainsi arrivé à quasiment annuler le principe du vote individuel.

A Lokossa, le problème rencontré par la population dans ses rapports avec l'administration locale n'est pas la rapidité du service, mais l'interaction avec une structure étrangère à ses utilisateurs. Le recours à une connaissance qui usera de sa proximité avec un membre de l'administration pour jouer un rôle d'intermédiaire (ce qui ne dispense cependant pas de "déposer la pierre" ${ }^{31}$ ) y est donc très répandu. Dans ce contexte, les habitants de Lokossa construisent l'ancrage des réseaux sociaux dans l'administration, au niveau des responsables administratifs élus (maires et chefs de quartier), sur une base collective et non pas individuelle ${ }^{32}$. Le cas de la commune de Ouèdèmè-Adja en est une belle illustration :

40 Alors que plusieurs candidats à la mairie de Ouèdèmè-Adja s'étaient déjà fait connaître, la population, n'étant satisfaite d'aucun d'entre eux, s'est concertée, sous la conduite de ses portes-parole légitimes, pour choisir son candidat. Une délégation des habitants de la commune, représentant les jeunes, les vieux et les associations locales, est allée solliciter la candidature de l'un de ses notables, un commerçant de près de 60 ans, ancien soldat de l'armée française et haut dignitaire de la section départementale du 'Christianisme céleste'. Après avoir hésité, celui-ci s'est décidé à se présenter parce qu'il est "un fils du coin et un patriote". Lors de l'élection, il l'a emporté très nettement 
sur les autres candidats qui n'ont obtenu que très peu de voix (à l'exception du candidat qu'il soutenait lui-même avant d'être sollicité par la population).

Dans d'autres communes, la population a tôt pris les choses en main en organisant une consultation populaire au cours de laquelle elle a débattu collectivement la question de savoir qui serait son candidat, celui à qui elle demanderait de se présenter et pour lequel elle voterait collectivement ${ }^{33}$. La décision ainsi prise n'était pas une consigne de vote à proprement parler (qui, dans le cas des maires, risquait d'ailleurs d'être mise à mal par le scrutin secret ${ }^{34}$ ), mais, en quelque sorte, le choix officiel de la population. Si elle n'a pas été strictement respectée, il n'en reste pas moins que, dans tous les cas recensés, le candidat désigné lors d'une telle réunion a toujours été élu haut la main.

Si tous les habitants sont conviés à de telles réunions, le poids des propos qui y sont tenus dépend fortement du statut de celui qui les émet ${ }^{35}$, Les femmes et les jeunes n'y sont pas censés prendre la parole, tandis que les aînés les plus riches en capital social influent fortement sur les débats, Le consensus qui en ressort est par conséquent fortement inspiré par ces derniers. Aussi a-t-on affaire à un processus électoral qui, s'il apparaît, dans sa phase finale, comme une élection où s'exprime une somme de choix individuels théoriquement libres de toute contrainte extérieure, entérine en réalité une sélection collective du maire, antérieure à l'élection à bulletins secrets organisée par l'État selon les normes du modèle démocratique occidental. Cette sélection repose sur un consensus construit selon des principes locaux, dont l'inégalité entre les voix des citoyens, à la différence du vote proposé par l'État, où toutes les voix sont supposées peser d'un poids égal ${ }^{36}$.

On est donc confronté à un rejet, de la part de la population, du mode de choix des élus locaux proposé par l'État. L'élection organisée par ce dernier n'est plus ici qu'un simulacre, qui a surtout valeur de test pour la capacité de la communauté à dicter son choix (ses logiques et ses normes) à l'ensemble des individus qui la composent.

Une volonté locale de consensus et de communication

Les principes qui motivent le choix d'un maire par la communauté sont essentiellement déterminés par les rôles que celle-ci voudrait le voir assumer. Deux d'entre eux retiendront ici notre attention, celui d'arbitre ou de gestionnaire des conflits locaux et celui de "courtier local de développement" (Olivier de Sardan 1996 : 102), par lesquels la population demande aux maires d'être des médiateurs entre le local et le global en assurant le lien entre la population et les institutions nationales voire internationales.

Le maire, comme d'ailleurs le chef de quartier, est très souvent sollicité pour arbitrer les conflits les plus divers. Qu'il s'agisse d'un conflit matrimonial ou d'une affaire de vol, on attend de lui que, tout en agissant au titre d'agent de l'État, il fasse prévaloir une gestion des conflits qui réponde aux normes locales et tienne notamment compte de leur proximité sociale avec les personnes concernées. La population appréhende en effet la gestion des conflits par l'État, qui est perçue comme distante, moins compréhensive ou moins soucieuse du contexte et parfois brutale. Le maire doit alors agir de manière à satisfaire, d'un côté, l'exigence de l'État d'une justice conforme à ses standards et, de l'autre, la demande populaire d'un règlement du conflit qui garantisse la paix et les liens sociaux qui unissent la communauté. Dans la mesure du possible, il tente cependant de se décharger au maximum de cette tâche difficile et de transférer la gestion des conflits aux autorités judiciaires.

Pour la population, le maire doit également être à même de créer une dynamique de développement local. Pour ce faire, il doit savoir interagir tant avec les instances 
supérieures de l'État et les organisations internationales de développement qu'avec les "fils du pays" installés dans les grandes villes ou à l'étranger, qui peuvent être de précieux relais auprès de celles-ci. Autrement dit, ses électeurs attendent de lui qu'il agisse comme leur "courtier local de développement". De ce point de vue, le degré d'instruction du représentant local et sa maîtrise de la langue française, gages d'une bonne communication avec l'extérieur, sont des déterminants importants du choix populaire; ils ne sont cependant pas absolus et le choix de la population, comme par exemple dans la commune de Ouèdèmè-Adja, à Lokossa, ne se porte pas nécessairement sur les candidat les plus instruits (enseignants retraités, directeurs d'école, etc.).

Ces deux rôles exigent donc des maires qu'ils sachent faire communiquer deux mondes socioculturels, celui de la localité et celui de l'extérieur (l'État, les acteurs internationaux de développement, etc.). La population prend clairement en compte cette dimension lors de l'élaboration de sa stratégie électorale, en cherchant à nommer à cette fonction des candidats "dotés de compétences permettant de (...) mettre en contact [ces deux mondes] et d'assurer la circulation de flux entre eux (flux symboliques ou politiques autant qu'économiques et matériels)" (Olivier de Sardan $1996: 102)^{37}$

Ces facettes du rôle des maires expliquent aussi pourquoi les Béninois sont très sensibles à la question de l'appartenance locale des représentants locaux de l'État. Ainsi, dans le cas de la commune de Ouèdèmè-Adja, la communauté a élu le candidat qui manifestait, outre la maîtrise du français et une certaine scolarisation, d'autres mérites tels que son "bon comportement", sa sobriété, ses moyens financiers ou sa mobilisation pour le développement local. Celles-ci marquent son bon enracinement dans la communauté, qualité importante aux yeux de la population, car pour être son intermédiaire auprès de l'extérieur, il faut non seulement savoir communiquer efficacement avec lui mais aussi être un porte-parole fidèle des aspirations de la communauté. L'enracinement local d'un représentant de l'État est ainsi un gage de proximité, il permet en outre à la population d'être en mesure de lui demander des comptes (Gbessèmèhlan \& Rijnierse 1995 : 71-2).

C'est avec la volonté de répondre à ce souci que l'État a nommé à la tête des circonscriptions urbaines des chefs originaires de celles-ci. Mais les espoirs qu'une telle mesure fit naître dans la population ont souvent été déçus. Les Béninois s'expliquent pourtant difficilement leur déception : un "fils du pays" aurait dû faire mieux. Il aurait dû manifester un souci aigu du développement local, que sa connaissance précise des besoins et du contexte locaux aurait permis de rendre efficace. Il aurait dû être plus proche de leurs réalités quotidiennes, communiquer avec ses concitoyens et partager leurs soucis. Si l'intensité de cette déception dépend évidemment du bilan personnel de chaque chef de circonscription, elle n'en est pas moins toujours présente à un degré ou un autre. La circonscription urbaine apparaît ainsi comme un lieu de rupture entre le citoyen et l'État: c'est le premier niveau de la hiérarchie administrative où le citoyen éprouve la distance qui le sépare de l'État ${ }^{38}$. Ce sentiment est notamment la conséquence d'un manque ou d'une absence de communication de la part de la circonscription qui fait que, si le maire (le niveau hiérarchique immédiatement inférieur) est perçu comme l'un des siens par la population, le chef de circonscription relève, quant à lui, davantage de l'ordre de l'altérité dans la perception populaire. Selon elle, il appartient bien plus à l'État qu'à sa communauté locale et il répond aux logiques du premier plutôt qu'à celles de la seconde. 

de circonscription, qui ne semble chercher ni à construire ni à s'appuyer sur un consensus local. Le maire et le chef de quartier, par contre, veillent, dans la mesure du possible, à prendre des décisions consensuelles. Cela est dû à leur situation, notamment à leur profond enracinement local et au fait qu'ils sont issus d'un choix collectif et public relevant lui-même d'une volonté de consensus. Il est inimaginable, pour les maires que nous avons rencontrés, de prendre une décision seul. Ils sont bien conscients du fait que leur autorité relève de la volonté populaire et ils veillent à ne pas se l'aliéner, ce qui n'est pas chose aisée tant les attentes de la population sont grandes, les moyens des mairies insignifiants et les incompréhensions faciles. C'est pourquoi la construction de consensus requiert une bonne communication. Si de ce point de vue tout le monde semble relativement satisfait au niveau du quartier ou de la commune, nous avons signalé qu'il en va tout autrement en ce qui concerne la circonscription urbaine.

51 Ces éléments nous permettent de mieux comprendre pourquoi la population place de nombreux espoirs en ses maires, qui, par leur proximité sociale, relèvent de son contrôle, et guère dans la circonscription qui lui échappe totalement. Ils nous éclairent aussi sur les raisons pour lesquelles les maires incarnent beaucoup moins que les chefs de circonscription les a priori populaires sur les hommes politiques, tels que leur désir d'enrichissement et de notoriété. représentants locaux de l'État avec la distinction entre représentants locaux élus par la population et ceux qui sont choisis par l'État. A la différence du maire et du chef de quartier, le chef de circonscription urbaine, nommé par le "haut", répond aux logiques $\mathrm{du}$ "haut" et n'arrive pas à combler le fossé qui le sépare du "bas" par une communication efficace. C'est bien là ce que lui reproche la population : apparaitre, peut-être par défaut de communication, comme un étranger répondant à des logiques externes.

Conclusions : de la décentralisation et de certaines de ses probables conséquences...

Ces premiers résultats de l'enquête menée conjointement par l'ENA et l'ULB nous ont permis de mettre en évidence que de nombreux Béninois restent mal ou sous-informés sur la décentralisation. Leur opinion en est donc mal fondée et repose surtout sur la confiance qu'ils ont en l'État. Leur crainte principale est que celle-ci soit l'occasion de leur abandon par l'État alors qu'ils ne voient pas, particulièrement en cette période économiquement difficile, comment ils pourraient être en mesure de supporter eux-mêmes le coût de la gestion des futures communes décentralisées.

Nous avons vu par ailleurs que la population s'adresse rarement à son administration. C'est l'obligation, qui lui est faite dans certaines circonstances, de disposer de documents officiels qui l'y contraint. D'une manière générale, on peut supposer qu'en renforçant l'autonomie de l'administration locale, la décentralisation accroîtra les rapports entre administrés et administration, et, par la même occasion, le recours aux diverses stratégies développées par les Béninois pour nouer ceux-ci d'une façon satisfaisante. Ces pratiques sont à la fois la conséquence et le signe de la distance qui sépare l'administration de ses administrés au Bénin. Elles visent tantôt à profiter de cette distance pour 'délocaliser' l'ancrage administratif des citoyens, tantôt à avoir prise sur cette administration pour réduire la distance qui la sépare de ses administrés.

Bulletin de l'APAD, 16 | 1998 

pluralité que les Béninois savent se rendre efficace. Par leur capacité à imposer, par des voies détournées, la multiplicité de leur territorialisation sociale à leur inscription territoriale administrative, les Béninois parviennent en effet à se trouver en position de choisir l'entité administrative avec laquelle ils doivent traiter indépendamment du lieu de leur résidence effective. Ce choix est déterminé par les performances relatives des différentes administrations (à ce propos, on pourrait dire que les Béninois ont en quelque sorte créé une "économie de marché" de l'administration) mais aussi par les caractéristiques de leur réseau personnel de relations avec l'appareil administratif. La décentralisation pourrait ainsi renforcer un usage de l'appareil d'État qui, même si ce n'est pas de la manière dont certains l'attendaient, n'en est pas moins doté d'une certaine dose d'empowerment et de philosophie néo-libérale. Il faut cependant souligner un effet pervers, du point de vue de l'État, de telles pratiques: le fait que la résidence effective ne corresponde pas à la résidence légale complique la tâche de l'État en matière de perception mais surtout de redistribution des contributions, ce qui peut s'avérer particulièrement important dans le cadre de la répartition des moyens financiers entre les entités décentralisées ${ }^{39}$.

D'autres stratégies, plus collectives, se fondent sur l'utilisation des élections pour ancrer les réseaux sociaux locaux dans l'administration afin de la rendre plus accessible. Ces stratégies ont permis de garantir une certaine proximité sociale des maires et chefs de quartier, aussi ne sont-ils pas perçus comme extérieurs à la communauté comme c'est le cas pour d'autres représentants de l'État et notamment le chef de circonscription.

57 A cet égard, le rôle des élections apparaît déterminant. En effet, les élections locales étant le moyen par lequel la population se concilie l'appareil administratif - ainsi qu'un moment $d$ "'inversion des rapports de force entre gouvernants et gouvernés" (Banégas 1998 : 79) à l'occasion duquel ces derniers doivent "rendre des comptes" à la population ${ }^{40}$-, on peut dire que le chef de circonscription échappe à la population parce qu'il n'est pas élu par elle. La décentralisation modifiera cette donne : le chef de circonscription urbaine ne sera plus choisi par l'État mais choisi en leur sein par les membres du conseil communal, qui sont eux directement élus par la population (Dossou 1997 : 16). Autrement dit, pour reprendre une terminologie chère à Jean-François Bayart, ils seront désormais issus du "bas" et non plus du "haut". décentralisation. Il faut en effet espérer que l'élection (quand bien même elle ne sera qu'indirecte) du chef de circonscription urbaine ${ }^{41}$ lui donnera un meilleur enracinement local et permettra d'instaurer les base d'une coopération solide, riche en communication et en consensus, avec la population, puisque désormais ce sera principalement sur elle que celui-ci devra compter pour mener à bien son action. 


\section{BIBLIOGRAPHIE}

Alber, E. 1997. "Le pouvoir local face aux mutations au niveau de l'État. Le cas d'un village bariba". Cahiers d'Etudes Africaines 145,37 (1) : 137-56.

Banegas, R. 1998. "Marchandisation du vote, citoyenneté et consolidation démocratique au Bénin". Politique Africaine 69 : 75-87.

Bayart, J.-F. 1992. "Les jalons d'une méthode". In : Bayart, J.-F.Mbembe, A. \& C. Toulabor (dir.) Le politique par le bas en Afrique noire. Contributions à une problématique de la démocratie, Paris, Karthala : 25-106.

Bierschenk, Th. et R. Mongbo. . "Le terroir en quête de démocratie". Politique Africaine 59 : 2-6.

Dossou, C. 1997. Décentralisation, déconcentration, découpage territorial. Ce qu'il faut savoir. Cotonou : Centre d'information et de documentation sur les collectivités locales au Bénin.

Gbessemehlan, V. \& E. Rijnierse. 1995. "Les élections en milieu rural : le cas de Ouessé". Politique Africaine $59: 70-81$.

Gupta, A. 1995. "Blurred boundaries : the discourse of corruption, the culture of politics and the imagined state". American Ethnologist 22 : 375-402.

Jaglin, S. \& A. Dubresson (die.) 1993. Pouvoirs et cités d'Afrique noire. Décentralisations en questions. Paris : Karthala.

Karlstrom, M. 1996. "Imagining democracy : political culture and democratisation in Buganda". Africa 66 (4) : 485-505.

Kearney, M. 1995. "The local and the global : the anthropology of globalization and transnationalism". Annual Review of Anthropology 24 : 547-65.

Olivier de Sardan, J.-P. 1996. "L'économie morale de la corruption en Afrique". Politique Africaine 63 : 97-116.

Séhouéto, L. 1992. "Présentation des résultats des enquêtes (RRA)". In : Décentralisation et démocratisation (doc. 2), Cotonou, Fondation Konrad Adernauer : 43-55.

Sinou, A. 1995. Le comptoir de Ouidah. Une ville africaine singulière. Paris : Karthala.

Spencer, J. 1997. "Post-colonialism and the political imagination". Journal of Royal Anthropological Institute 3 (1) : 1-19.

Sperber, D. 1996. La contagion des idées. Paris : Odile Jacob.

\section{NOTES}

1.Le fait que la Cour constitutionnelle vient de juger non conformes à la constitution du Bénin plus de 80 articles des 5 lois votées par les députés qui devaient fixer le cadre juridique de ta décentralisation (La Région, mai 1998, n 10;8) ne constitue que le dernier en date des multiples obstacles qui jalonnent sa mise en place.

2.Ce projet, qui bénéficie d'un financement de l'Agence Générale de Coopération au Développement (AGCD) de Belgique, a débuté en juin 1997.

3.Parfois appelées sous-préfectures. 
4.Un questionnaire fermé a été administré aux agents municipaux tandis que les élus locaux et les administrés ont été interrogés, dans la langue locale, sur base d'un guide d'entretien ou, dans certains cas, d'un questionnaire se mi-ouvert. La présente étude se base essentiellement sur les réponses de ces deux dernières catégories d'informateurs. L'échantillon pour ces différentes localités était de 102 administrés et 29 élus locaux à Bohicon, 101 administrés el 40 élus locaux à Lokossa, 75 administrés et 30 élus locaux à Ouidah. On a veillé a interrogé des administrés d'âge et de sexe différents, issus des différentes catégories socioprofessionnelles (néanmoins, les hommes interrogés sont toujours deux à trois fois plus nombreux que les femmes).

5.De nombreux projets ont pour objectif d'accompagner la mise en place du processus de décentralisation au Bénin. Ils se consacrent notamment à la production de données nécessaires pour l'élaboration de la décentralisation, à la récolte d'informations issues du haut (notamment une revue de presse) ou à la réalisation de formations destinées à ceux qui auront à gérer la décentralisation au plus haut niveau local (futurs responsables et secrétaires généraux des entités décentralisées). Le projet conjoint de l'ENA et de l'ULB leur est complémentaire en ce qu'il vise, quant à lui, une approche par le bas de l'administration locale, au travers de ses usagers et des cadres des niveaux les plus bas de la hiérarchie administrative. Se reporter à Bayart (1992) pour le cadre théorique d'une telle approche en Afrique.

6.Il résulte de nos enquêtes que, si l'on excepte le crieur public, la radio est le média qui bénéficie de la plus grande audience auprès des populations ; c'est aussi celui qui couvre l'ensemble du pays, qui est le plus facile d'accès (il ne nécessite pas d'être alphabétisé) et qui est aussi, à terme. le moins onéreux.

7.Voir notamment C. Agossa, "Décentralisation au Bénin. Les populations cherchent à comprendre". La Région, mai 1998, n¹0: 7.

8.Lorsque j'emploie l'expression 'les Béninois', il faut comprendre les Béninois interrogés. Ceux-ci sont considérés comme représentatifs des habitants de leur circonscription urbaine.

9.Ce processus a été mis en évidence par Dan Sperber (1996). Quand un individu ne peut déterminer la légitimité d'une information en fonction de son contenu, il le fait en fonction de la légitimité qu'il reconnaît il l'émetteur de cette information. Autrement dit, si l'émetteur est légitime, l'information l'est aussi.

10.20 ans. interrogée par Carmen Ahéhéhinnou à Avlékété (Ouidah) le 26 juin 1997. 11.On noiera qu'en ce qui concerne l'adduction d'eau et l'électrification, les populations demanderesses se sont révélées capables de mener à bien des souscriptions portant sur des montants relativement importants compte tenu de leur niveau de vie fort modeste, et couvrant tantôt la totalité des frais, tantôt la partie à cofinancer par la population (l'autre partie pouvant être apportée par la société para-étatique concernée ou par une instance de l'Etat). Malheureusement, il est arrivé plusieurs fois que les attentes qui avaient donné lieu à ces souscriptions ne soient pas satisfaites. les promesses à l'origine de ces attentes ayant été soit excessives, voire fallacieuses, soit mal comprises par la population.

12. Cette enquête s'est déroulée dans diverses localités de 4 des 6 départements actuels du Bénin (dont Lokossa qui fait aussi partie de l'échantillon de l'enquête ENA-ULB). 13.Il est possible que cette divergence soit due à des différences méthodologiques entre les deux enquêtes.

14.Le fait qu'une majorité de Béninois soit dépourvue de carte d'identité ou en possède une qui est périmée (la durée de validité de celle-ci étant de 5 ans). alors qu'il s'agit là 
du principal motif pour lequel les Béninois s'adressent à leur administration locale, illustre bien la paucité des contact entre celte dernière et la population.

15.On remarquera que de ce point de vue l'Etat représenté par son administration auto-justifie sa présence : il est là pour fournir un service qu'il a lui-même rendu nécessaire.

16.En témoigne le fait que la plupart de nos interlocuteurs estiment que c'est le désir de "bouffer".

autrement dit le désir d'enrichissement, qui a motivé les candidats aux responsabilités administratives locales à se présenter.

17.Notons que certains Béninois ne se sont jamais adressés à une administration tandis que d'autres. notables ou personnes ressources, sont plus ou moins régulièrement. Selon la personnalité du responsable administratif. appelés à participer à des réunions de consultation.

18.10.000 francs CFA par mois pour un maire et 5.000 pour un chef de quartier.

19.Il faut préciser. à la décharge des maires, que le volume de visites d'une commune n'est pas très élevé.

20.Cette exigence de rapidité est propre à Ouidah ; à Lokossa et Bohicon, cette revendication est nettement secondaire.

21.De leur propre initiative ou suite à une "suggestion" du fonctionnaire, selon les cas. Cette pratique est parfois transformée au détriment de l'usager : certains fonctionnaires font comprendre qu'à défaut de "déposer la pierre" la procédure sera allongée.

22.Cette stratégie peut servir soit un objectif de rapidité, soit un objectif de moindre coût, soit les deux (gratuité du 'service rapide' ou gratuité totale). En effet, une relation peut toujours vous délivrer l'acte gratuitement ou à moindre coût, particulièrement si c'est un maire ou un chef de quartier, du fait du contrôle moindre qui s'exerce sur ceux-ci par comparaison avec les agents de la circonscription urbaine, surveillés plus directement par leurs supérieurs hiérarchiques.

23.Il faut préciser qu'un certain nombre de Béninois n'ont fait la connaissance du chef de leur quartier que lorsqu'ils ont eu quelque chose à lui demander.

24.C'est particulièrement vrai pour les jeunes gens.

25.Cette particularité explique, au moins en partie, que cette stratégie soit surtout usitée à Ouidah et Cotonou, où les structures de transport et la mobilité sont bonnes et où les Béninois peuvent tabler sur un grand nombre d'instances administratives locales de même niveau, mais peu à Lokossa ou à Bohicon.

26.Il est des maires qui ont en permanence le cachet communal sur eux de façon à ce qu'on puisse les solliciter en toute occasion.

27.Voir Alber (1997 : 148-50) pour le cas d'un village bariba dans le nord-est du Bénin. 28.On a néanmoins procédé occasionnellement à un nouveau vote là où un élu de 1991 n'était plus en mesure d'assurer sa fonction (généralement en raison d'un décès).

29.Elle n'est pas non plus spécifique au Bénin et on peut supposer qu'un tel facteur détermine très souvent un vote pour une part plus ou moins grande, qu'il ait lieu en Europe, en Afrique ou ailleurs dans le monde.

30.En particulier dans ses communes les plus rurales comme Houin ou Ouèdèmè-Adja.

31.Davantage, et apparemment essentiellement suite à une demande d'un agent de l'Etat, pour éviter un allongement de la procédure que pour permettre son raccourcissement. 
32. De ce point de vue, Bohicon est assez comparable à Lokossa et E. Alber renseigne le même procédé dans un village bariba du nord du Bénin : "suite à une pression massive et sous la convocation de l'autorité symbolique du tebosuno [le détenteur du pouvoir traditionnel] eut lieu une assemblée du village qui élit Saka Bio, le candidat autour duquel une entente se scella d'avance" (Alber 1997 : 150).

33. Cette pratique explique pourquoi il est rare qu'un habitant de Lokossa s'adresse à une administration autre que la sienne.

34.Les élections communales se font à bulletins secrets ; chaque candidat est associé à un pictogramme (la représentation d'un fruit, d'un animal, etc.) et l'ensemble des sigles est remis à chaque électeur qui dépose dans l'urne celui du candidat de son choix. 35.On retrouve ici la logique qui préside également à la légitimation des propos de l'Etat sur la décentralisation parce qu'il en est l'émetteur et qu'il est lui-même légitime ; seulement, à la différence de la décentralisation pour laquelle il n'y a pas de discours concurrentiel, l'Etat (et avec lui son discours) souffre ici d'un crédit moindre par rapport à la communauté locale.

36.Pour une autre discussion des principes d'élection et de sélection, voir Karlstrôm (1996 : 497-8) qui renseigne le cas de la population de l'ancien royaume du Buganda (dans l'Ouganda actuel) qui marque. elle, sur base de ses valeurs locales, sa préférence pour le principe de l'élection proposé aujourd'hui par l'Etat par comparaison avec celui de sélection qui préside au choix des autorités locales dans le système traditionnel local.

37. Ces médiations partent autant du haut que du bas : si le niveau local cherche à placer ses médiateurs dans la sphère étatique (et n'est-ce pas là la quintessence du principe de l'élection par le peuple des représentants de l'Etat), l'inverse est tout aussi vrai : l'Etat central essaye lui aussi d'ancrer ses courtiers politiques au niveau local - au Bénin, c'était particulièrement vrai durant le régime marxiste-léniniste de Kérékou I (Bierschenk \& Mongbo 1995 : 4).

38.Pour les niveaux supérieurs comme celui du parlement, voir Gbessèmèhlan \& Rijnierse (1995 : 71-2).

39.Cela influera aussi sur le nombre de conseillers communaux à élire puisqu'il dépend du nombre d'habitants recensés dans l'entité (Dossou 1997 : 19).

40.Beaucoup des Béninois que nous avons interrogés voyaient dans les prochaines élections locales (qui auraient dû se tenir il a deux ans déjà) l'occasion de signifier aux élus actuels ce qu'ils pensent d'eux.

41. Qui portera alors le titre de maire.

\section{AUTEUR}

\section{CHRISTOPHE ANTHOINE}

Centre d'Anthropologie Culturelle de l'Université Libre de Bruxelles, 44, avenue Jeanne, 1050 Bruxelles, Belgique. Tél. +32-(2) 6503396 Fax. +32-(2) 6504337 -

canthoin@ulb.ac.be 